\title{
Henry E. Sigerist-Preis 1971
}

(Dr. Markus-Guggenheim-Schnurr-Stiftung für Geschichte der Medizin und der Naturwissenschaften)

Der Jury, bestehend aus den Herren PD Dr. Haffter (Basel), Dr.Gubser (Zürich) und als Vorsitzendem Prof. Dr. Hintzsche (Bern), lagen in diesem Jahre 15 Arbeiten zur Beurteilung vor. Sie zeichnete von diesen zwei ex aequo mit dem Henry-E.-Sigerist-Preis aus. Wie in solchem Falle üblich stehen die Namen in alphabetischer Reihe:

Frau Dr. Ursula Bueck-Rich, Limmattalstraße 33, 8049 Zürich, wurde der Preis zugesprochen für ihre Dissertation Ernst Heinrich Weber (1795-1878) und der Anfang einer Physiologie der Hautsinne. Die Wichtigkeit der von Weber mit einfachen Hilfsmitteln durchgeführten Experimente für eine allgemeine Sinnesphysiologie wird betont und seine Bedeutung als eines der Begründer der physikalisch orientierten Physiologie hervorgehoben. Die in der Hauptsache aus lateinischer Primärliteratur erarbeitete Abhandlung fand Anerkennung wegen ihrer klaren Formulierung und der exakten Begriffsdefinitionen.

Bei der Beurteilung der Dissertation von Herrn Dr. Fritz Kubli, Limmattalstraße 285, 8049 Zürich, ließ sich die Jury durch Fachleute beraten. Mit diesen anerkennt sie, daß die Studie über Louis de Broglie und die Entdeckung der Materiewelle eines Preises würdig ist. Die bisher fehlende kritisch-geschichtliche Beurteilung des Beitrages, den de Broglie zur Entwicklung der Quantenmechanik leistete, ist im Sachverhalt richtig wiedergegeben; sie hat zu wesentlichen neuen Gesichtspunkten geführt. Sowohl Inhalt als Literaturbearbeitung wurden als sehr gut bewertet. Da Gespräche mit Herrn de Broglie die Hauptquelle der Abhandlung von Herrn Dr. Kubli waren, erforderte die Darstellung viel Takt und Geschick, was gleichfalls anerkannt wird.

Da auch im vergangenen Jahre der Usus befolgt wurde, weitere zur Spitzengruppe Gehörende hier zu nennen, werden noch drei Namen in alphabetischer Reihe angeführt, nämlich die der Herren Dr. Paul Johann Dubler mit einer Dissertation über Johann Jakob Baader 1810-1879, Arzt und Politiker in Gelterkinden, Dr. Albert Erlanger, dessen Dissertationsthema lautete: Der Psychiater Rudolf Leubuscher 1821-1861 und schließlich Dr. Avinoam Safran mit seiner Dissertation: La contribution du Judaïsme au développement de la médecine.

E. Hintzsche 